\title{
An EPAS1 Haplotype Is Associated With High Altitude Polycythemia in Male Han Chinese at the Qinghai-Tibetan Plateau
}

\author{
Yu Chen, MD; Chunhua Jiang, MD; Yongjun Luo, MD; Fuyu Liu, MD; Yuqi Gao, MD \\ From the Department of Pathophysiology and High Altitude Physiology (Drs Chen, Jiang, Liu, and Gao), and Department of High Altitude Disease \\ (Dr Luo), College of High Altitude Military Medicine, and Key Laboratory of High Altitude Medicine, Third Military Medical University, Ministry of \\ Education, and Key Laboratory of High Altitude Medicine, People's Liberation Army (Drs Chen, Jiang, Luo, Liu, and Gao), Chongqing, China.
}

\begin{abstract}
Background.-Hemoglobin concentration at high altitude is considered an important marker of high altitude adaptation, and native Tibetans in the Qinghai-Tibetan plateau show lower hemoglobin concentrations than Han people who have emigrated from plains areas. Genetic studies revealed that EPAS1 plays a key role in high altitude adaptation and is associated with the low hemoglobin concentration in Tibetans. Three single nucleotide polymorphisms (rs13419896, rs4953354, rs 1868092) of noncoding regions in EPAS1 exhibited significantly different allele frequencies in the Tibetan and Han populations and were associated with low hemoglobin concentrations in Tibetans.

Methods. - To explore the hereditary basis of high altitude polycythemia (HAPC) and investigate the association between EPAS1 and HAPC in the Han population, these 3 single nucleotide polymorphisms were assessed in 318 male Han Chinese HAPC patients and 316 control subjects. Genotyping was performed by high resolution melting curve analysis.

Results.-The G-G-G haplotype of rs13419896, rs4953354, and rs1868092 was significantly more frequent in HAPC patients than in control subjects, whereas no differences in the allele or genotype frequencies of the 3 single nucleotide polymorphisms were found between HAPC patients and control subjects. Moreover, genotypes of rs1868092 (AA) and rs4953354 (GG) that were not observed in the Chinese Han in the Beijing population were found at frequencies of $1.6 \%$ and $0.9 \%$, respectively, in our study population of HAPC patients and control subjects.

Conclusions.-Carriers of this EPAS1 haplotype (G-G-G, rs13419896, rs4953354, and rs1868092) may have a higher risk for HAPC. These results may contribute to a better understanding of the pathogenesis of HAPC in the Han population.
\end{abstract}

Key words: high altitude polycythemia, EPAS1, high resolution melting, Qinghai-Tibetan plateau

\section{Introduction}

The Qinghai-Tibetan plateau covers a vast area with a harsh natural environment, and millions of people live in this region. Approximately 12 million people were reported to permanently reside in the Qinghai-Tibetan plateau in 2006, and this number increases every year; most of the increase in population has come from Han people emigrating from low altitude areas. ${ }^{1}$ For low altitude populations who move to high altitude areas, hemoglobin $(\mathrm{Hb})$ concentration increases at a certain range because of the hypoxic environment, and this response is

Corresponding author: Yuqi Gao, MD, College of High Altitude Military Medicine, Third Military Medical University, Chongqing 400038, China (e-mail: gaoy66@yahoo.com). crucial for them to acclimatize to high altitude. As they stay longer at high altitude, some people, especially Han, are prone to chronic mountain sickness, which is characterized by symptoms of long-term hypoxia. ${ }^{2,3}$ The most striking of these features is excessive erythropoiesis, also called high altitude polycythemia (HAPC). ${ }^{3}$ Although testosterone level, ${ }^{4-6}$, sleep quality, ${ }^{7,8}$ oxidative stress, and immune response ${ }^{10}$ are involved in the pathogenesis of HAPC, the genetic basis of HAPC has not been studied extensively, ${ }^{11,12}$ especially in the Han population.

Of all human populations worldwide, native Tibetans of the Qinghai-Tibetan plateau are regarded as the one best adapted to high altitudes, and they exhibit lower $\mathrm{Hb}$ concentrations than Han who have emigrated from low altitudes, even those who have acclimated to the high 
altitude. $^{13}$ This phenomenon is considered largely genetic. Moreover, the incidence of HAPC among Tibetans is lower than that among Han, ${ }^{14}$ and mounting evidence suggests that genetic factors contribute to the development of altitude-related illnesses. ${ }^{15-17}$ Recently, significant progress has been made in the study of the genetic basis of high altitude adaptation in Tibetans, and recent research has highlighted a new aspect of the genetic basis of HAPC. Namely, peroxisome proliferatoractivated receptor $\alpha$ (PPARA), ${ }^{18}$ egl nine homolog 1 (EGLN1), ${ }^{18-22}$ and endothelial Per-Arnt-Sim (PAS) domain protein 1 (EPAS1) $)^{19-24}$ have been reported to play important roles in high altitude adaptation in Tibetans. Of these 3 candidate genes, EPAS1 has been implicated as making the greatest contribution to genetic adaptation to high altitude and to the low $\mathrm{Hb}$ concentrations observed in the Tibetan population. ${ }^{23,24}$

Expression of EPAS1 is limited to organs that are involved in oxygen transport and metabolism, such as the lung, placenta, and vascular endothelium, ${ }^{25}$ and it also contributes to many biological processes and diseases related to metabolism, ${ }^{26}$ angiogenesis, ${ }^{27,28}$ inflammation, ${ }^{29,30}$ and cancer, ${ }^{31-33}$ indicating that EPAS1 plays a key role in oxygen sensing and metabolism. Moreover, it was also found that EPAS1 was associated with high altitude pulmonary edema, ${ }^{17}$ which is an idiopathic disease of high altitude ${ }^{34}$ in the Han population. Genetic studies of high altitude adaptation in Tibetans suggest that EPAS1 has been subjected to strong natural selection by the high altitude environment. Noncoding DNA sequences in or near EPAS1 are significantly different in the Tibetan and Han populations, and these regions are associated with low $\mathrm{Hb}$ concentration in Tibetans. ${ }^{23,24}$

Three single nucleotide polymorphisms (SNPs) in noncoding regions_-rs13419896, rs4953354, and rs1868092_ not only show significant differences in frequencies between the Tibetan and Han populations, ${ }^{20}$ but also are associated with low $\mathrm{Hb}$ in Tibetans. ${ }^{24}$ To better understand the relationship between EPAS1 and HAPC in the Han population, we analyzed the genotypes and allele frequencies of the 3 SNPs, rs13419896 (A/G), rs4953354 (A/G), and rs1868092 (A/G), using the high resolution melting (HRM) method ${ }^{35,36}$ in male HAPC patients and control subjects from the Han population in the Qinghai-Tibetan plateau.

\section{Methods}

\section{STUDY GROUPS}

In all, 318 HAPC patients and 316 healthy control subjects were recruited from the Han population at the Qinghai-Tibetan plateau in China. All patients and control subjects who participated in this research had lived at an altitude above $4000 \mathrm{~m}$ for at least 3 months. According to the standard diagnostic criteria for chronic mountain sickness, we selected male HAPC patients with excessive polycythemia ( $\mathrm{Hb} \geq 210 \mathrm{~g} / \mathrm{L})$ and without chronic pulmonary diseases, which are the same as the inclusion criteria for chronic mountain sickness. ${ }^{37}$ Characteristics of all participants are listed in Table 1. This research was approved by the Ethics Committee of the Third Military Medical University of China.

\section{ALLELES FREQUENCIES OF TIBETAN AND CHINESE HAN BEIJING POPULATION}

Alleles frequencies of rs13419896, rs4953354, and rs1868092 in the Tibetan population were obtained from Peng et al. ${ }^{20}$ Genotypes and alleles of these 3 SNPs in the Chinese Han Beijing population were downloaded from HapMap (http://hapmap.ncbi.nlm.nih.gov/cgi-perl/ gbrowse/hapmap28_B36/; HapMap data rel28 phaseII+ III,August 10, on NCBI B36 assembly, dbSNP b126). These data were also included in further analysis.

Table 1. Characteristics of healthy control subjects and high altitude polycythemia patients

\begin{tabular}{|c|c|c|c|c|c|}
\hline Characteristics & $n$ & Controls & $n$ & HAPC & $P$ value \\
\hline Age, years & 315 & $25.50 \pm 6.50(18-46)$ & 280 & $24.73 \pm 5.58(18-51)$ & .192 \\
\hline Hemoglobin, g/L & 316 & $181.08 \pm 10.48(150-203)$ & 318 & $221.97 \pm 11.3(210-282.5)$ & $<.001$ \\
\hline $\mathrm{SaO}_{2}, \%$ & 255 & $89.43 \pm 3.19(53-95)$ & 243 & $87.90 \pm 3.52(70-97)$ & $<.001$ \\
\hline Heart rate, beats/min & 255 & $82.52 \pm 13.63(52-146)$ & 243 & $88.15 \pm 16.38(58-144)$ & $<.001$ \\
\hline $\mathrm{SBP}, \mathrm{mm} \mathrm{Hg}$ & 255 & $123.42 \pm 11.89(90-158)$ & 241 & $124.56 \pm 12.79(92-176)$ & .307 \\
\hline $\mathrm{DBP}, \mathrm{mm} \mathrm{Hg}$ & 255 & $74.15 \pm 10.07(52-115)$ & 241 & $77.76 \pm 11.18(51-124)$ & $<.001$ \\
\hline \multirow{2}{*}{ Smoking status } & 159 & Yes & 141 & Yes & \multirow{2}{*}{.435} \\
\hline & 102 & No & 78 & No & \\
\hline \multirow{2}{*}{ Drinking status } & 26 & Yes & 23 & Yes & \multirow{2}{*}{.582} \\
\hline & 202 & No & 151 & No & \\
\hline
\end{tabular}

Values are mean \pm SD (minimum-maximum). Comparisons used unpaired Student's $t$ test.

HAPC, high altitude polycythemia; SBP, systolic blood pressure; DBP, diastolic blood pressure. 
Table 2. Information on 3 genotyped single nucleotide polymorphisms

\begin{tabular}{|c|c|c|c|c|c|}
\hline SNP No. & NCBI rs & Chromosome position & Location & Base change & $M A F^{a}$ \\
\hline 1 & rs13419896 & chr2:46556345 & Intron1 & $\mathrm{A} / \mathrm{G}$ & 0.303 \\
\hline 2 & rs4953354 & chr2:46575388 & Intron2 & $\mathrm{A} / \mathrm{G}$ & 0.133 \\
\hline 3 & rs1868092 & chr2:46614202 & $3^{\prime}$ intergenic region to EPAS1 & $\mathrm{A} / \mathrm{G}$ & 0.073 \\
\hline
\end{tabular}

\footnotetext{
${ }^{a}$ Minor allele frequencies (MAF) of Chinese Han Beijing in HapMap release no. 28 data. (http://hapmap.ncbi.nlm.nih.gov/cgi-perl/gbrowse/ hapmap28_B36/; HapMapDataRel28PhaseII_III,August10,on NCBI B36 assembly,dbSNP b126).

SNP, single nucleotide polymorphism; NCBI, National Center for Biotechnology Information.
}

\section{DNA EXTRACTION}

Peripheral venous blood $(2 \mathrm{~mL})$ was collected from HAPC patients and healthy control subjects in ethylenediaminetetraacetic acid anticoagulation tubes and kept at $-80^{\circ} \mathrm{C}$ until use. Genomic DNA was extracted from $250 \mu \mathrm{L}$ whole blood using the Omega (Omega BioTek, Norcross, GA) DNA extraction kit according to the manufacturer's protocol. A NanoDrop ND-1000 spectrophotometer (Isogen Life Science, IJsselstein, Netherlands) was used to measure the quality and concentration of the extracted DNA. Then, the primary extracted DNA was diluted to a final concentration of $20 \mathrm{ng} / \mu \mathrm{L}$ in the elution buffer provided in the Omega DNA extraction kit.

\section{GENOTYPING}

The 3 SNPs (Table 2) were genotyped using a HRM assay in the Rotor-Gene Q real-time polymerase chain reaction (PCR) system (Qiagen, Valencia, CA). ${ }^{38}$ The primer sequences used for PCR are listed in Table 3. The PCR was performed in $25-\mu \mathrm{L}$ reactions. The reaction mixture contained $1 \mu \mathrm{L}$ DNA (20 ng), $2.5 \mu \mathrm{L}$ PCR buffer with $\mathrm{Mg}^{2+}$ (Takara Bio, Shiga, Japan), $2 \mu \mathrm{L}$ dNTPs $(200 \mu \mathrm{M}), 1 \mu \mathrm{L}$ forward primer $(10 \mu \mathrm{M}), 1 \mu \mathrm{L}$ reverse primer $(10 \mu \mathrm{M}), 1 \mu \mathrm{L}$ EvaGreen (Biotium, Hayward, CA), $0.125 \mu \mathrm{L}$ TaKaRa Taq DNA polymerase (5 U/ $\mu \mathrm{L})$, and $16.375 \mu \mathrm{L}$ sterilized deionized distilled water $\left(\mathrm{ddH}_{2} \mathrm{O}\right)$. The PCR was carried out in a PTC-200 DNA Engine thermal cycler (Bio-Rad, Hercules, CA) with a protocol of $95^{\circ} \mathrm{C}$ for 5 minutes; 35 cycles of
5 seconds at $95^{\circ} \mathrm{C}, 30$ seconds at $60^{\circ} \mathrm{C}$, and 30 seconds at $72^{\circ} \mathrm{C}$; and a final hold at $72^{\circ} \mathrm{C}$ for 3 minutes.

After amplification, the PCR tubes were transferred to the Rotor-Gene Q real-time PCR system and subjected to a melting protocol (temperature increased from $80^{\circ} \mathrm{C}$ to $90^{\circ} \mathrm{C}$ at $0.1^{\circ} \mathrm{C} / 2 \mathrm{~s}$ ). The HRM curve analysis was carried out using the Rotor-Gene Q 2.0.3 software, and genotypes were identified by melting curve differences.

\section{DNA SEQUENCING}

After genotyping, we randomly sequenced 63 subjects (10\% of all samples), including examples of each genotype from both HAPC patients and controls, to assure the accuracy of the HRM curve analysis. Theses samples were sent to BGI (Wuhan, China) for DNA sequencing.

\section{STATISTICAL ANALYSIS}

The data are shown as the mean \pm SD. Hardy-Weinberg equilibrium as well as genotypes and alleles distributions between HAPC and controls were tested by chi-square test using SPSS version 13.0 software (SPSS, Chicago, IL). The odds ratio, 95\% confidence interval, and $P$ value were calculated between HAPC patients and controls, $P<.05$ was considered to indicate a significant difference. Haplotype analysis was performed using SHEsis online software (http://analysis.bio-X.cn) ${ }^{39,40}$ The haplotype analysis on SHEsis was carried out by a $2-x-5$ contingency table test using a chi-square test with 4 degrees of freedom, for 5 haplotypes included in this

Table 3. Sequences of the primers used to amplify the 3 single nucleotide polymorphism regions

\begin{tabular}{lllc}
\hline & Primer & \multicolumn{1}{c}{ Sequence $\left(5^{\prime}-3^{\prime}\right)$} & Product (base pair) \\
\hline \multirow{2}{*}{ rs13419896 } & Forward & AAACCCTTCCTGGTTGAGTA & 84 \\
& Reverse & CAGATGACAATCCCTGAACC & 88 \\
rs1868092 & Forward & CTGTTCTGTATATTCACATAGTGC & \\
& Reverse & GCCTCCAAATTGAAGTCCTA & 97 \\
rs4953354 & Forward & TAGGCCTAAAGAGGCGAAAT & \\
& Reverse & GCCATTGTCTTGCATTGTTC & \\
& & & \\
\hline
\end{tabular}


Table 4. Distribution of rs13419896, rs4953354, and rs1868092 genotypes in high altitude polycythemia patients and control subjects

\begin{tabular}{|c|c|c|c|c|}
\hline$S N P$ & Genotype & $H A P C$ & Control & $P$ value \\
\hline \multirow[t]{3}{*}{ rs13419896 } & AA & $31(9.7)$ & $30(9.5)$ & \multirow[t]{3}{*}{$.34\left(\chi^{2} 2.15\right.$, df 2$)$} \\
\hline & $\mathrm{AG}$ & $136(42.8)$ & $153(48.4)$ & \\
\hline & GG & $151(47.5)$ & $133(42.1)$ & \\
\hline \multirow[t]{3}{*}{ rs1868092 } & AA & $4(1.3)$ & $6(1.9)$ & \multirow[t]{3}{*}{$.27\left(\chi^{2} 2.65\right.$, df 2$)$} \\
\hline & $\mathrm{AG}$ & $43(13.5)$ & $56(17.7)$ & \\
\hline & GG & $271(85.2)$ & $254(80.4)$ & \\
\hline \multirow[t]{3}{*}{ rs4953354 } & GG & $3(0.9)$ & $3(0.9)$ & \multirow[t]{3}{*}{$.64\left(\chi^{2} 0.90\right.$, df 2$)$} \\
\hline & $\mathrm{AG}$ & 88 (27.7) & 77 (24.4) & \\
\hline & $\mathrm{AA}$ & $227(71.4)$ & $236(74.7)$ & \\
\hline
\end{tabular}

Values are n (\%). Comparison of genotypes frequencies of rs13419896, rs4953354, and rs1868092 in high altitude polycythemia (HAPC) patients and control subjects were made by chi-square test.

SNP, single nucleotide polymorphism; df, degrees of freedom.

analysis. A Bonferroni correction was used for genetic tests; the threshold for significance was 0.0045 at the significance level of 0.05 . Table 4 shows 3 comparisons, Table 5 shows 3 comparisons, and Table 6 shows 5 comparisons; the total comparisons of 11 times meant that the genetic tests (genotypes, alleles, and haplotypes) would be judged significant only if the tests yielded a $P$ value $<.0045$.

\section{Results}

\section{HARDY-WEINBERG EQUILIBRIUM TESTS AND DISTRIBUTION OF GENOTYPES AND ALLELES}

Significant differences were observed between the melting curves of each genotype of each SNP, allowing us to easily distinguish the genotypes (Figure 1). The measured genotypes frequencies are presented in Table 4. All 3 SNPs were in Hardy-Weinberg equilibrium in control group rs13419896 (0.137), rs4953354 (0.170), and rs1868092 (0.227); and in HAPC patients rs13419896
(0.963), rs4953354 (0.079), and rs1868092 (0.137). We also compared these SNP data to those from the Chinese Han Beijing population of the HapMap project (release \#28 data) and to Tibetan population data from a previous report. ${ }^{20}$ Single site association analyses revealed no significant differences in the genotype (Table 4) or allele (Table 5) frequencies between the HAPC patients and controls in our study. The allele frequencies in our data were not significantly different from those published for the Chinese Han Beijing, even though several genotypes in our data were not found in the Chinese Han Beijing data, and there was a significant difference between our data and the data from the Tibetan population (Table 7). Moreover, our genotyping results indicated that persons with higher $\mathrm{Hb}$ concentrations may carry the genotype that is considered advantageous in the Tibetan population. For instance, the A allele and AA genotype of rs1868092 are believed to be adaptive in Tibetans, ${ }^{20}$ whereas our data show that distribution of the AA genotype of rs 1868092 between HAPC patients and controls has no significant difference.

Table 5. Comparisons of alleles from high altitude polycythemia patients and control subjects

\begin{tabular}{|c|c|c|c|c|}
\hline SNP & HAPC & Control & $P$ value & OR $(95 \% C I)$ \\
\hline \multicolumn{5}{|c|}{ rs13419896 } \\
\hline A & $198(31.1)$ & $213(33.7)$ & \multirow{2}{*}{$.33\left(\chi^{2} 0.96\right.$, df 1$)$} & Reference \\
\hline G & $438(68.9)$ & $419(66.3)$ & & $0.89(0.70-1.13)$ \\
\hline \multicolumn{5}{|c|}{ rs 1868092} \\
\hline A & $51(8.0)$ & $68(10.8)$ & \multirow{2}{*}{$.09\left(\chi^{2} 2.80\right.$, df 1$)$} & Reference \\
\hline G & $585(92.0)$ & $564(89.2)$ & & $0.72(0.49-1.06)$ \\
\hline \multicolumn{5}{|c|}{ rs4953354 } \\
\hline A & $542(85.2)$ & $549(86.9)$ & \multirow{2}{*}{$.40\left(\chi^{2} 0.72\right.$, df 1$)$} & Reference \\
\hline G & $94(14.8)$ & $83(13.1)$ & & $0.87(0.63-1.20)$ \\
\hline
\end{tabular}

Values are $\mathrm{n}(\%)$. Comparison of alleles frequencies of rs13419896, rs4953354, and rs1868092 in high altitude polycythemia (HAPC) patients and control subjects were made by chi-square test.

SNP, single nucleotide polymorphism; OR, odds ratio; CI, confidence interval. 
Table 6. Haplotype analysis for rs13419896, rs4953354, rs 1868092 in high altitude polycythemia patients and control subjects

\begin{tabular}{lcclll}
\hline & HAPC & Control $n(\%)$ & $\chi^{2}$ & P value & OR (95\% CI) \\
\hline A-G-A & $27.69(4.4)$ & $29.69(4.7)$ & 0.154 & .695 & $0.899(0.529-1.529)$ \\
G-A-A & $144.51(22.7)$ & $144.09(22.8)$ & 0.078 & .780 & $0.963(0.739-1.255)$ \\
G-A-G & $30.25(4.8)$ & $35.46(5.6)$ & 0.628 & .428 & $0.818(0.497-1.346)$ \\
G-G-A & $357.02(56.1)$ & $358.07(56.7)$ & 0.52 & .471 & $0.919(0.730-1.156)$ \\
G-G-G & $53.22(8.4)$ & $26.38(4.2)$ & 8.806 & $.003^{a}$ & $2.046(1.264-3.309)$ \\
\hline
\end{tabular}

Values are $\mathrm{n}(\%)$.

${ }^{a}$ Bonferroni-corrected $P \leq .033$ (11 tests), comparison of G-G-G haplotype vs others in high altitude polycythemia (HAPC) patients and control subjects.

\section{HAPLOTYPE ANALYSIS}

Based on our data, we constructed haplotypes using SHEsis online software, and haplotypes with frequencies below 0.03 were excluded from further analysis. The SNP order of haolotype was rs13419896, followed by

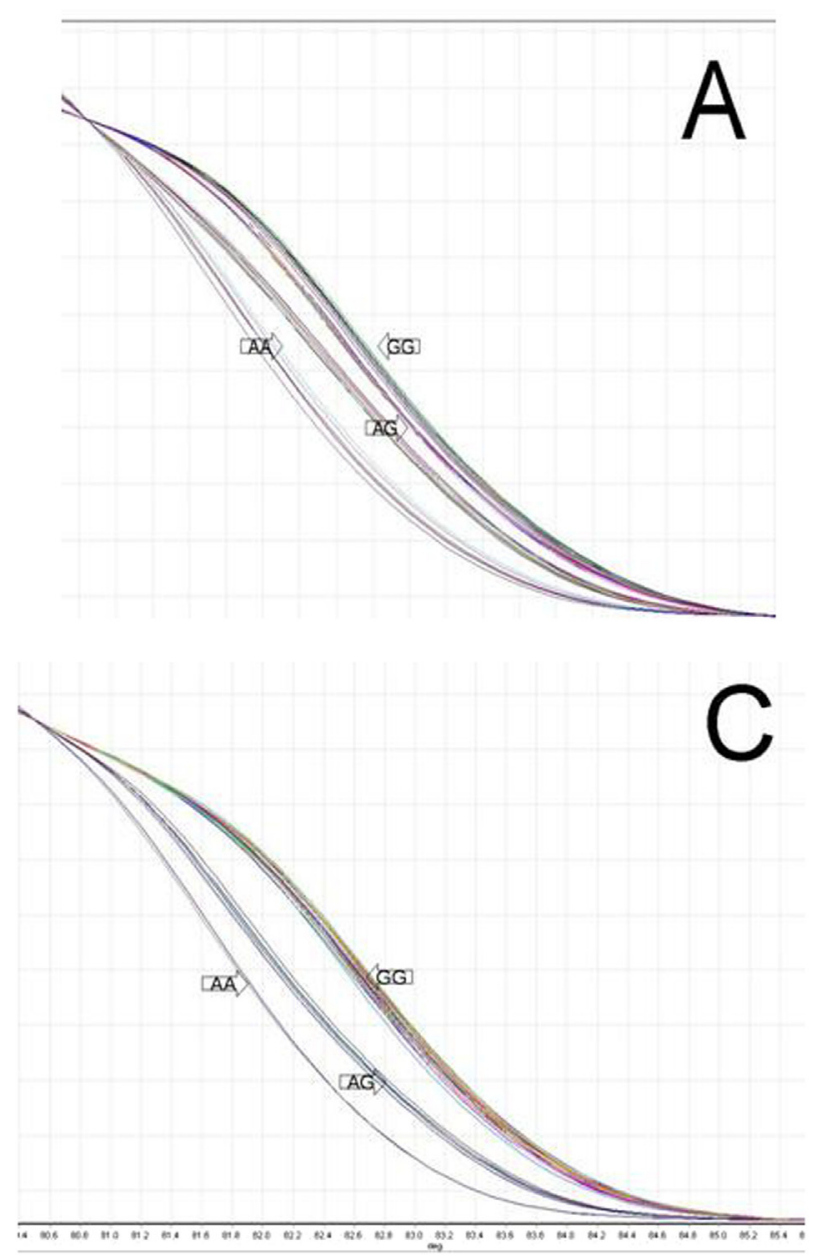

rs4953354 and rs1868092. These results indicated that the frequency of the G-G-G haplotype was significantly higher in the HAPC group than in the control group $(P=$ .003 ; odds ratio 2.046 ; $95 \%$ confidence interval: 1.264 to 3.309), whereas other haplotypes showed no significant

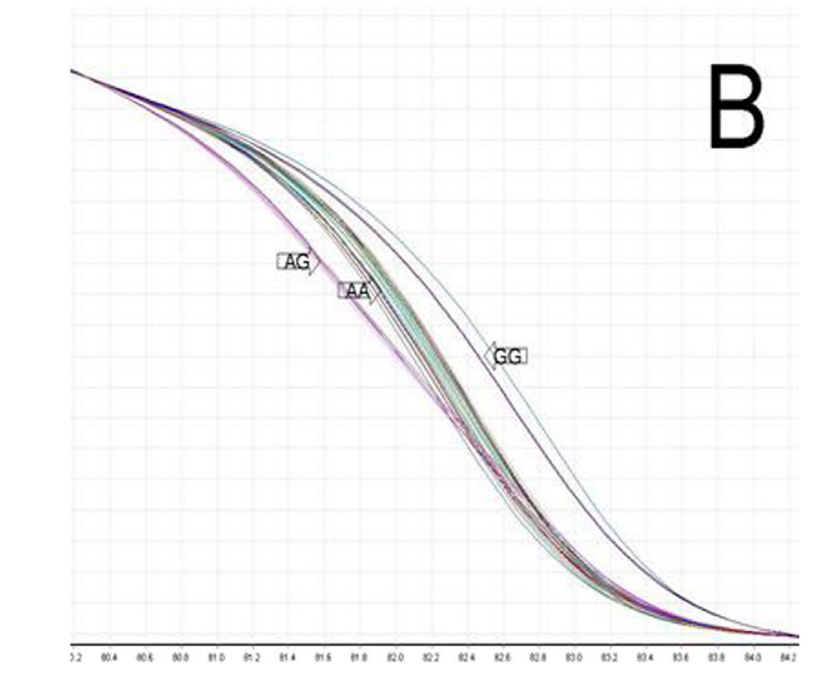

Figure 1. Melting curves analysis for the identification of genotypes in 3 single nucleotide polymorphisms: (A) rs13419896, (B) rs4953354, and (C) $\mathrm{rs} 1868092$. 
Table 7. Distributions of allele and genotype frequencies in the Tibetan, Chinese Han Beijing (HapMap), and Han (current study) populations

\begin{tabular}{|c|c|c|c|}
\hline$S N P$ & $\begin{array}{c}\text { Tibetan } \\
\text { (allele: \%) }\end{array}$ & $\begin{array}{c}C H B \\
\text { (genotype: \%) }\end{array}$ & $\begin{array}{c}\text { Han } \\
\text { (genotype: \%) }\end{array}$ \\
\hline \multirow[t]{5}{*}{ rs13419896 } & & AA: 7.3 & AA: 9.6 \\
\hline & & AG: 46.0 & AG: 45.6 \\
\hline & & GG: 46.7 & GG: 44.8 \\
\hline & $\mathrm{A}: 76.3^{a}$ & A: 30.3 & A: 32.2 \\
\hline & $\mathrm{G}: 23.7^{a}$ & G: 69.7 & G: 67.8 \\
\hline \multirow[t]{5}{*}{ rs1868092 } & & AA: $0^{b}$ & AA: 1.6 \\
\hline & & AG: 14.6 & AG: 15.6 \\
\hline & & GG: 85.4 & GG: 82.8 \\
\hline & A: $69.8^{a}$ & A: 7.3 & A: 9.4 \\
\hline & $\mathrm{G}: 30.2^{a}$ & G: 92.7 & G: 90.6 \\
\hline \multirow[t]{5}{*}{ rs4953354 } & & AA: 73.3 & AA: 72.6 \\
\hline & & AG: 26.7 & AG: 26.0 \\
\hline & & GG: $0^{b}$ & GG: 1.4 \\
\hline & $\mathrm{A}: 26.9^{a}$ & A: 86.7 & A: 86.0 \\
\hline & $\mathrm{G}: 73.1^{a}$ & G: 13.3 & $\mathrm{G}: 14.0$ \\
\hline
\end{tabular}

The allele frequencies of the 3 single nucleotide polymorphisms (SNPs) in the Tibetan population were derived from the averages reported by Peng et al. ${ }^{20}$ The Chinese Han Beijing (CHB) data were downloaded from HapMap (http://hapmap.ncbi.nlm.nih.gov/cgi-perl/ gbrowse/hapmap28_B36/; HapMapDataRel28PhaseII_III,August10,on NCBI B36 assembly,dbSNP b126). Comparison of alleles between Tibetan, Chinese Han Beijing and Han were made by chi-square test.

${ }^{a}$ Not reported in Chinese Han Beijing.

${ }^{b} P<.01$ between Tibetan and Chinese Han Beijing; $P<.01$ between Tibetan and Han.

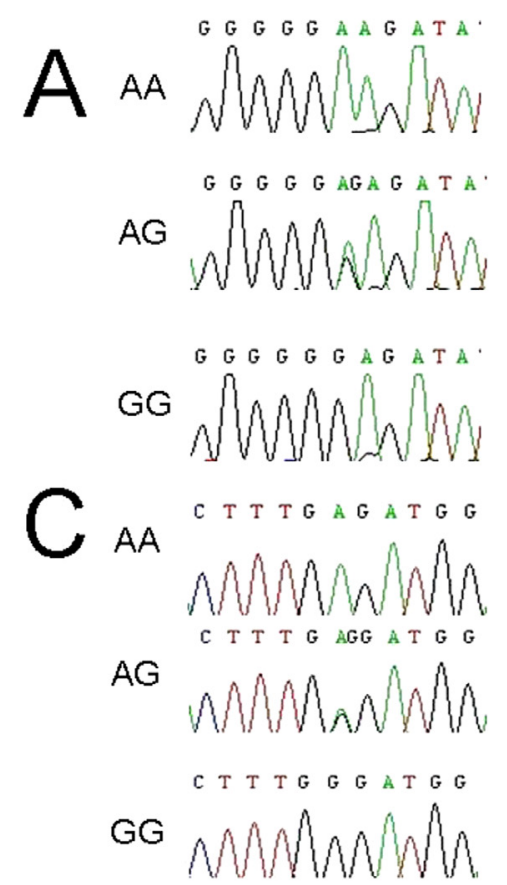

difference in frequency between the control and HAPC groups (Table 6).

\section{DNA SEQUENCING CONFIRMED THE ACCURACY OF THE HRM ANALYSIS}

Results of DNA sequencing were in accordance with the genotypes determined by HRM curve analysis in all samples sequenced, as well as rare genotypes of rs1868092 (A/A) and rs4953354 (G/G) in the Han population (Figure 2), indicating that both the sensitivity and the specificity of the HRM curve analysis in the present study were $100 \%$. The frequencies of the 2 rare genotypes rs1868092 (A/A) and rs4953354 (G/G) were $1.6 \%$ and $0.9 \%$, respectively, in our sample.

\section{Discussion}

This study revealed an association between SNPs in EPAS1 and HAPC in male Han subjects. Although significant differences in the alleles and genotypes frequencies of the 3 SNPs (rs13419896, rs4953354, and rs1868092) of EPAS1 were observed between the Tibetan and Han populations, our results showed no differences in the distribution of genotypes or alleles of the 3 SNPs between HAPC patients and control subjects from the Han population. However, haplotype analysis indicated that the G-G-G (rs13419896, rs4953354, and rs1868092) haplotype was more frequent in the HAPC patients compared with the control subjects.

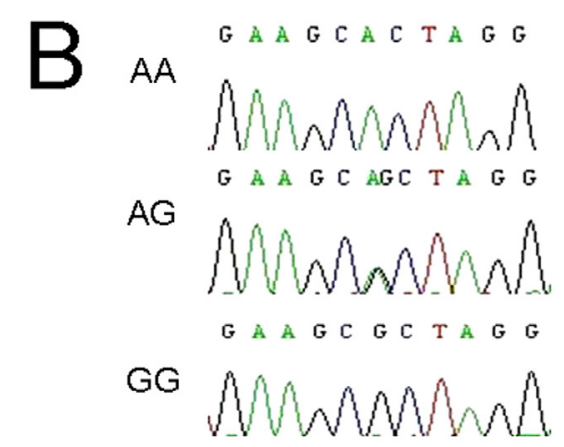

Figure 2. Genotypes (AA, AG, and GG) identified by the direct DNA sequencing data: (A) rs13419896, (B) rs4953354, and (C) rs1868092. 
The EPAS1 gene lies on chromosome 2p21-p16 and is involved in multiple biological processes associated with oxygen sensing and metabolism. As a transcription factor, EPAS1 functions as targeting its downstream genes, such as iron absorption genes, which is essential for erythropoieses. ${ }^{41}$ It is also reported that mutation of EPAS1 exons would cause excessive erythrocytosis under normoxic conditions. ${ }^{42-45}$ Moreover, a polymorphism in the intron of EPAS1chr2:46441523(hg18) was associated with chronic mountain sickness in Tibetans, ${ }^{46}$ and this polymorphism also showed great allele frequency differences between Tibetan and Han populations. $^{23}$

However, even though DNA sequences of EPAS1 showed significant differences between Tibetan and Han populations, it is unclear whether expression or function of EPAS1 differs between these 2 groups, and the role of $\mathrm{Hb}$ in high altitude adaptation is still under discussion. ${ }^{47}$ This study leads us to reconsider the role that EPAS1 plays in HAPC in the Han population and in high altitude adaptation in the Tibetan population.

First, because the genotypes and alleles frequencies of the 3 SNPs showed no differences between HAPC and controls, it appears that the $\mathrm{Hb}$ concentration in the Han population at high altitude is not so greatly associated with SNPs as is reported in the Tibetan population. ${ }^{24}$ One possible explanation for this phenomenon is that Tibetans have experienced thousands of years of selective pressure by high altitude hypoxia. ${ }^{19-24,48}$ EPAS1 exhibits the strongest signatures of natural selection and might be expressed in the context of a unique genetic model in the Tibetan population in comparison with the Han population; this context could affect its downstream gene expression to result in the low $\mathrm{Hb}$ concentrations observed in Tibetans. Because the Han population has not undergone such a long-term adaptation, however, the genetic model of EPAS1 in the Han population is adapted to normoxia; therefore, the $\mathrm{Hb}$ concentration in the Han population may not be so highly associated with a single SNP variant of EPAS1 when they emigrate from plains areas to high altitude, although it is still higher than that in Tibetans. Thus, even HAPC patients in the Han population may carry the genotype that is adaptive in the Tibetan background.

Second, we found that the frequency of the G-G-G haplotype was significantly higher in HAPC patients than in control subjects. We speculate that this haplotype might induce splice site variants ${ }^{49}$ or affect microRNA regulation, ${ }^{50}$ which could change the expression level of EPAS1 target genes or influence the gene-gene interaction, leading to the development of HAPC at high altitude. The effect of EPAS1 on iron absorptionassociated genes in the small intestine ${ }^{41}$ suggests a potential mechanism of HAPC; namely, a high altitude environment stabilizes EPAS1 structure, and EPAS1 functions in the intestine to promote more proteins involved in iron absorption synthesis encourage excessive iron absorption, eventually leading to the occurrence of HAPC. However, the G-G-G haplotype is rare in the Han population, indicating that this genetic change is not sufficient to explain the entire pathogenesis of HAPC in the Han population.

Third, it remains unclear whether the differences in EPAS1 between Tibetan and Han populations are the results of natural selection by high altitude or simply the differences between these 2 populations without affects of high altitude. Finally, more studies are needed to determine whether $\mathrm{Hb}$ concentration is really the ideal marker of high altitude adaptation. ${ }^{47}$

We also identified genotypes that were not observed in the HapMap Chinese Han Beijing database, rs4953354 (GG) and rs1868092(AA) (Figure 2). Although this study revealed no different distributions of the alleles or genotype frequencies of these 3 SNPs between HAPC patients and healthy controls, further investigations should focus on finding more variants in or near EPAS1 and other genes that are associated with high altitude adaptation and erythropoiesis. Moreover, we should also consider the effects of epigenetic modifications of EPAS1, such as DNA methylation and microRNA regulation, on the pathogenesis of HAPC.

In conclusion, this study indicates that a variation of EPAS1 may be involved in the genetic susceptibility to HAPC in the Han population; and the G-G-G haplotype constructed by rs13419896, rs4953354, and rs 1868092 in EPAS1 may increase the risk of HAPC in the male Han population.

\section{Acknowledgments}

This work was supported by the National Key Technology Research and Development Program of China (2009BAI85B01) and the National Basic Research Program of China (973 Program; 2012CB518201). We would like to express our appreciation to those who collected samples in the Qinghai-Tibetan plateau, and we thank everyone who participated in this study.

\section{References}

1. Wu T, Kayser B. High altitude adaptation in Tibetans. High Alt Med Biol. 2006;7:193-208.

2. Xing G, Qualls C, Huicho L, et al. Adaptation and maladaptation to ambient hypoxia; Andean, Ethiopian and Himalayan patterns. PLoS One. 2008;3:e2342. 
3. Reeves JT, Leon-Velarde F. Chronic mountain sickness: recent studies of the relationship between hemoglobin concentration and oxygen transport. High Alt Med Biol. 2004;5:147-155.

4. Gonzales GF, Tapia V, Gasco M, et al. Serum testosterone levels and score of chronic mountain sickness in Peruvian men natives at 4340 m. Andrologia. 2011;43:189-195.

5. Gonzales GF, Tapia V, Gasco M, et al. High serum zinc and serum testosterone levels were associated with excessive erythrocytosis in men at high altitudes. Endocrine. 2011;40:472-480.

6. Gonzales GF, Gasco M, Tapia V, et al. High serum testosterone levels are associated with excessive erythrocytosis of chronic mountain sickness in men. Am J Physiol Endocr Metab. 2009;296:E1319-E1325.

7. Kong FY, Li Q, Liu SX. Poor sleep quality predicts decreased cognitive function independently of chronic mountain sickness score in young soldiers with polycythemia stationed in Tibet. High Alt Med Biol. 2011;12: 237-242.

8. Spicuzza L, Casiraghi N, Gamboa A, et al. Sleep-related hypoxaemia and excessive erythrocytosis in Andean highaltitude natives. Eur Respir J. 2004;23:41-46.

9. Jefferson JA, Simon J, Escudero E, et al. Increased oxidative stress following acute and chronic high altitude exposure. High Alt Med Biol. 2004;5:61-69.

10. Jiang C, Liu FY, Luo YJ, et al. Gene expression profiling of high altitude polycythemia in Han Chinese migrating to the Qinghai-Tibetan plateau. Mol Med Rep. 2012;5:287293.

11. Mejia OM, Prchal JT, Leon-Velarde F, et al. Genetic association analysis of chronic mountain sickness in an Andean high-altitude population. Haematologica. 2005; 90:13-19.

12. Leon-Velarde F, Mejia O. Gene expression in chronic high altitude diseases. High Alt Med Biol. 2008;9:130-139.

13. Wu T, Wang $X$, Wei $C$, et al. Hemoglobin levels in Qinghai-Tibet: different effects of gender for Tibetans vs. Han. J Appl Physiol. 2005;98:598-604.

14. Wu TY. Chronic mountain sickness on the QinghaiTibetan plateau. Chin Med J (Engl). 2005;118:161-168.

15. Rupert JL, Koehle MS. Evidence for a genetic basis for altitude-related illness. High Alt Med Biol. 2006;7:150-167.

16. Stobdan T, Karar J, Pasha MA. High altitude adaptation: genetic perspectives. High Alt Med Biol. 2008;9:140-147.

17. Yang YZ, Wang YP, Qi YJ, et al. Endothelial PAS domain protein $1 \mathrm{Chr} 2: 46441523$ (hg18) polymorphism is associated with susceptibility to high altitude pulmonary edema in Han Chinese. Wilderness Environ Med. 2013;24:315-320.

18. Simonson TS, Yang Y, Huff CD, et al. Genetic evidence for high-altitude adaptation in Tibet. Science. 2010;329: 72-75.

19. Bigham A, Bauchet M, Pinto D, et al. Identifying signatures of natural selection in Tibetan and Andean populations using dense genome scan data. PLoS Genet. 2010;6: e1001116.
20. Peng Y, Yang Z, Zhang H, et al. Genetic variations in Tibetan populations and high-altitude adaptation at the Himalayas. Mol Biol Evol. 2011;28:1075-1081.

21. $\mathrm{Xu} \mathrm{S}, \mathrm{Li} \mathrm{S}$, Yang $\mathrm{Y}$, et al. A genome-wide search for signals of high-altitude adaptation in Tibetans. Mol Biol Evol. 2011;28:1003-1011.

22. Wang B, Zhang YB, Zhang F, et al. On the origin of Tibetans and their genetic basis in adapting high-altitude environments. PLoS One. 2011;6:e17002.

23. Yi X, Liang Y, Huerta-Sanchez E, et al. Sequencing of 50 human exomes reveals adaptation to high altitude. Science. 2010;329:75-78.

24. Beall CM, Cavalleri GL, Deng L, et al. Natural selection on EPAS1 (HIF2alpha) associated with low hemoglobin concentration in Tibetan highlanders. Proc Natl Acad Sci USA. 2010;107:11459-11464.

25. Semenza GL. Life with oxygen. Science. 2007;318:62-64.

26. Majmundar AJ, Wong WJ, Simon MC. Hypoxia-inducible factors and the response to hypoxic stress. Mol Cell. 2010;40:294-309.

27. Skuli N, Simon MC. HIF-1alpha versus HIF-2alpha in endothelial cells and vascular functions: is there a master in angiogenesis regulation? Cell Cycle. 2009;8:3252-3253.

28. Skuli N, Liu L, Runge A, et al. Endothelial deletion of hypoxia-inducible factor-2alpha (HIF-2alpha) alters vascular function and tumor angiogenesis. Blood. 2009;114: 469-477.

29. Semenza GL. Oxygen sensing, homeostasis, and disease. N Engl J Med. 2011;365:537-547.

30. Eltzschig HK, Carmeliet P. Hypoxia and inflammation. N Engl J Med. 2011;364:656-665.

31. Kaelin WG. The von Hippel-Lindau tumour suppressor protein: O2 sensing and cancer. Nat Rev Cancer. 2008;8:865-873.

32. Han SS, Yeager M, Moore LE, et al. The chromosome 2 p21 region harbors a complex genetic architecture for association with risk for renal cell carcinoma. Hum Mol Genet. 2012;21:1190-1200.

33. Xue X, Taylor M, Anderson E, et al. Hypoxia-inducible factor-2alpha activation promotes colorectal cancer progression by dysregulating iron homeostasis. Cancer Res. 2012;72:2285-2293.

34. Scherrer U, Rexhaj E, Jayet PY, et al. New insights in the pathogenesis of high-altitude pulmonary edema. Prog Cardiovasc Dis. 2010;52:485-492.

35. Reed GH, Kent JO, Wittwer CT, High-resolution DNA. melting analysis for simple and efficient molecular diagnostics. Pharmacogenomics. 2007;8:597-608.

36. Vandersteen JG, Bayrak-Toydemir P, Palais RA, et al. Identifying common genetic variants by high-resolution melting. Clin Chem. 2007;53:1191-1198.

37. Leon-Velarde F, Maggiorini M, Reeves JT, et al. Consensus statement on chronic and subacute high altitude diseases. High Alt Med Biol. 2005;6:147-157.

38. Wu Z, Yuan H, Zhang X, et al. Development and interlaboratory validation of unlabeled probe melting curve analysis for detection of JAK2 V617F mutation in polycythemia vera. PLoS One. 2011;6:e26534. 
39. Li Z, Zhang Z, He Z, et al. A partition-ligationcombination-subdivision EM algorithm for haplotype inference with multiallelic markers: update of the SHEsis. Cell Res. 2009;19:519-523. http://analysis.bio-x.cn.

40. Shi YY, He L. SHEsis, a powerful software platform for analyses of linkage disequilibrium, haplotype construction, and genetic association at polymorphism loci. Cell Res. 2005;15:97-98.

41. Anderson ER, Xue X, Shah YM. Intestinal hypoxiainducible factor-2alpha (HIF-2alpha) is critical for efficient erythropoiesis. J Biol Chem. 2011;286:19533-19540.

42. Percy MJ, Chung YJ, Harrison C, et al. Two new mutations in the HIF2A gene associated with erythrocytosis. Am J Hematol. 2012;87:439-442.

43. Van Wijk R, Sutherland S, Van Wesel AC, et al. Erythrocytosis associated with a novel missense mutation in the HIF2A gene. Haematologica. 2010;95:829-832.

44. Percy MJ, Furlow PW, Lucas GS, et al. A gain-offunction mutation in the HIF2A gene in familial erythrocytosis. N Engl J Med. 2008;358:162-168.

45. Martini M, Teofili L, Cenci T, et al. A novel heterozygous HIF2AM535I mutation reinforces the role of oxygen sensing pathway disturbances in the pathogenesis of familial erythrocytosis. Haematologica. 2008;93:10681071.

46. Buroker NE, Ning XH, Zhou ZN, et al. EPAS1 and EGLN1 associations with high altitude sickness in Han and Tibetan Chinese at the Qinghai-Tibetan Plateau. Blood Cells Mol Dis. 2012;49:67-73.

47. Wilson MJ, Julian CG, Roach RC. Genomic analysis of high altitude adaptation: innovations and implications. Curr Sports Med Rep. 2011;10:59-61.

48. Zhao M, Kong QP, Wang HW, et al. Mitochondrial genome evidence reveals successful Late Paleolithic settlement on the Tibetan Plateau. Proc Natl Acad Sci USA. 2009;106:21230-21235.

49. Antonacopoulou AG, Floratou K, Bravou V, et al. The survivin-31 snp in human colorectal cancer correlates with survivin splice variant expression and improved overall survival. Cell Oncol (Dordr). 2011;34:381-391.

50. Gong J, Tong Y, Zhang HM, et al. Genome-wide identification of SNPs in microRNA genes and the SNP effects on microRNA target binding and biogenesis. Hum Mutat. 2012;33:254-263. 